\title{
Validation of a Candidate Deoxynivalenol-Inactivating UDP-Glucosyltransferase from Barley by Heterologous Expression in Yeast
}

\author{
Wolfgang Schweiger, ${ }^{1}$ Jayanand Boddu, ${ }^{2}$ Sanghyun Shin, ${ }^{2}$ Brigitte Poppenberger, ${ }^{1}$ Franz Berthiller, ${ }^{3}$ \\ Marc Lemmens, ${ }^{4}$ Gary J. Muehlbauer, ${ }^{2}$ and Gerhard Adam ${ }^{1}$ \\ ${ }^{1}$ Department of Applied Genetics and Cell Biology, University of Natural Resources and Applied Life Sciences, A-1160 \\ Vienna, Austria; ${ }^{2}$ Department of Agronomy and Plant Genetics, University of Minnesota, St. Paul 55108, U.S.A.; ${ }^{3}$ Center for \\ Analytical Chemistry, Department of Agrobiotechnology (IFA-Tulln), University of Natural Resources and Applied Life \\ Sciences, A-3430 Tulln, Austria; ${ }^{4}$ Institute of Biotechnology in Plant Production, Department of Agrobiotechnology (IFA- \\ Tulln), University of Natural Resources and Applied Life Sciences, A-3430 Tulln, Austria
}

Submitted 6 November 2009. Accepted 9 March 2010.

\begin{abstract}
Resistance to the virulence factor deoxynivalenol (DON) due to formation of DON-3-O-glucoside (D3G) is considered to be an important component of resistance against Fusarium spp. which produce this toxin. Multiple candidate UDP-glycosyltransferase (UGT) genes from different crop plants that are either induced by Fusarium spp. or differentially expressed in cultivars varying in Fusarium disease resistance have been described. However, UGT are encoded by a very large gene family in plants. The study of candidate plant UGT is highly warranted because of the potential relevance for developing Fusarium-spp.-resistant crops. We tested Arabidopsis thaliana genes closely related to a previously identified DON-glucosyltransferase gene by heterologous expression in yeast and showed that gene products with very high sequence similarity can have pronounced differences in detoxification capabilities. We also tested four candidate barley glucosyltransferases, which are highly DON inducible. Upon heterologous expression of full-length cDNAs, only one gene, HvUGT13248, conferred DON resistance. The conjugate D3G accumulated in the supernatant of DON-treated yeast transformants. We also present evidence that the product of the TaUGT3 gene recently proposed to encode a DON-detoxification enzyme of wheat does not protect yeast against DON.
\end{abstract}

The trichothecene mycotoxin deoxynivalenol (DON) is produced by a complex of Fusarium spp., including Fusarium graminearum and the species recently separated from it (Starkey et al. 2007), F. culmorum, F. pseudograminearum, and others. These pathogens can cause important agricultural diseases such as Fusarium head blight (scab) and crown rot of wheat and barley, as well as ear and stalk rot of maize (Vigier et al. 2001; Logrieco et al. 2002; Leonard and Bushnell 2003; Desjardins 2006). Fusarium diseases of cereals (Goswami and Kistler 2004; Foroud and Eudes 2009) are economically highly

Corresponding author: G. Adam; Telephone: 0043-1-47654-6380; Fax: 0043-1-47654-6392; E-mail: gerhard.adam@boku.ac.at

Current address of B. Poppenberger: Max F. Perutz Laboratories, University of Vienna, A-1030 Vienna, Austria.

* The $e$-Xtra logo stands for "electronic extra" and indicates that two supplementary tables are published online. relevant and can lead to widespread contamination of food with the mycotoxin DON (Schothorst and van Egmond 2004). Maximum tolerated limits for DON in food commodities (European Commission 2006a, 2007) and guideline levels for animal feed (European Commission 2006b) have been established in the European Union to prevent health risks to consumers.

DON acts as a protein biosynthesis inhibitor and is a virulence factor of $F$. graminearum, which facilitates spread of the pathogen and contributes to disease symptoms (Proctor et al. 1995; Bai et al. 2001; McCormick 2003). The effect of the loss of trichothecene production due to inactivation of the gene encoding trichodiene synthase (TRI5), the first step in DON biosynthesis, is variable and depends on the host plant species and genotype. In wheat, DON suppresses the formation of a cell-wall-based barrier; thus, tri5 mutants (trichothecene nonproducing) exhibit little spread from the initial infection site to other spikelets (Jansen et al. 2005). Barley exhibits higher spreading resistance than wheat and the effect of the loss of DON production is less pronounced (Maier et al. 2006; Boddu et al. 2007) and most likely also variable between cultivars. Because the protein biosynthesis inhibitor DON could suppress the translation of transcripts induced during plant defense, toxin resistance is considered to be a component of the complex polygenic and quantitative trait of Fusarium disease resistance (Buerstmayr et al. 2009). In hexaploid wheat, the major quantitative trait loci for Fusarium spp. spreading resistance was found to co-localize with resistance to the development of bleaching symptoms caused by application of DON to wheat ears (Lemmens et al. 2005). The mechanism causing high toxin resistance was shown to be the higher ability to convert DON into DON-3-O-glucoside (D3G) (Lemmens et al. 2005). This mechanism of DON metabolism was first described for a maize suspension culture (Sewald et al. 1992). The glucose-conjugate D3G exhibited a dramatically reduced ability to inhibit protein synthesis of wheat ribosomes in vitro (Poppenberger et al. 2003). Also, barley can form D3G upon treatment with DON (Gardiner et al. 2010). The ability to detoxify DON in barley could be responsible for the comparatively high spreading resistance. Recently, data showing the occurrence of D3G in naturally infected small-grain cereals and maize have become available (Berthiller et al. 2005; Sasanya et al. 2008). The molar ratio of $\mathrm{D} 3 \mathrm{G} / \mathrm{DON}$ in grain is, on average, approximately $15 \%$, reaching levels of up $46 \%$ (Berthiller et al. 2009a). The glucoside is 
also formed in Fusarium spp.-contaminated barley grains during the steeping process (germination) of malting (Lancova et al. 2008), and D3G concentrations in beer reach or exceed the level of DON (Kostelanska et al. 2009).

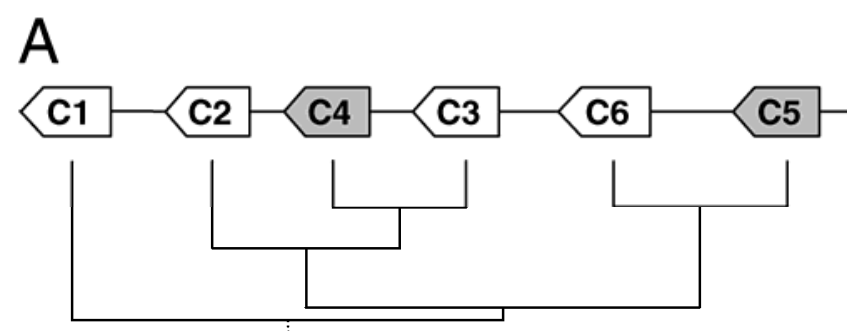

$\mathrm{B}$

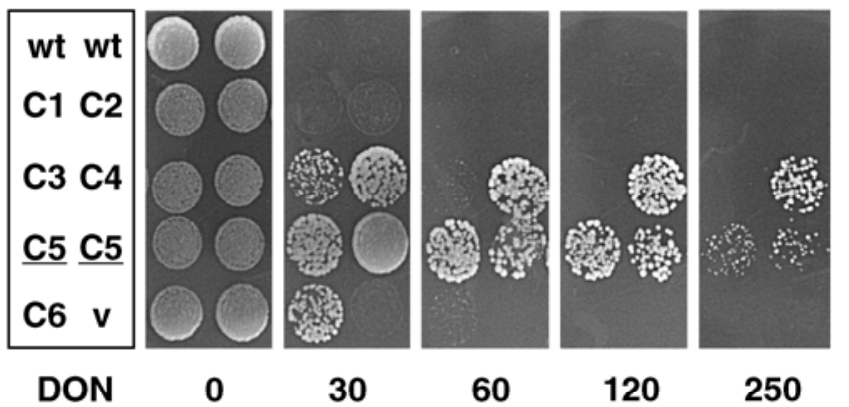

C

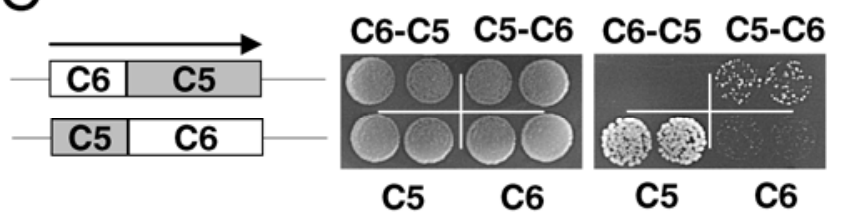

DON [mg/l] $0 \quad 60$
$\mathrm{D}$

\section{UGT73C5 (=DOGT1) UGT73C6 (inactive) UGT73C4 (active) UBQ5}

Fig. 1. Characterization of the Arabidopsis thaliana UGT73C gene cluster. A, Schematic representation of the chromosomal organisation and phylogenetic tree of the UGT73C gene cluster on chromosome II of Arabidopsis. $(U G T 73 C 1=\mathrm{At} 2 \mathrm{~g} 36750, U G T 73 C 2=\mathrm{Atg} 236760, U G T 73 C 4=\operatorname{Atg} 236770$, $U G T 73 C 3=\operatorname{Atg} 236780, U G T 73 C 6=\operatorname{Atg} 236790$, and $U G T 73 C 5=D O G T 1=$ Atg236800). Genes providing deoxynivalenol (DON) protection in yeast with DON at $120 \mathrm{mg} /$ liter are shaded in gray. B, DON resistance of yeast transformants containing the $U G T 73 C$ expression vectors spotted on YPD plates with the indicated concentration of DON (mg/liter). C1 = abbreviation of $U G T 73 C 1$, and so on; wt = untransformed wild-type strain YZGA515; v = transformed with empty vector (YZBP65). C, DON resistance of yeast strains (spotted in duplicate) expressing the parental $U G T 73 C 5$ (C5) or $U G T 73 C 6$ (C6) in the lower row or hybrid genes (upper row). Hybrid designation C5-C6 corresponds to the gene with the $\mathrm{N}$ terminus from $\mathrm{C} 5$ and the $\mathrm{C}$ terminus of C6. D, Reverse-transcription polymerase chain reaction analysis of induction of selected UGT73 gene family members in Arabidopsis thaliana after treatment with DON at $5 \mathrm{mg} / \mathrm{liter}$. Loading control UBQ5, 25 cycles, incubation time with DON given in hours. Designation inactive or active refers to the resistance phenotype conferred by the genes in yeast against DON at $120 \mathrm{mg} / \mathrm{liter}$.
A glucosyltransferase gene from Arabidopsis thaliana encoding an enzyme with the ability to convert DON into D3G had been cloned based on the DON resistance phenotype conferred by the respective cDNA in a toxin-sensitive yeast strain (Poppenberger et al. 2003). The Arabidopsis DON-glucosyltransferase $(D O G T 1=U G T 73 C 5)$ is located in a cluster of six paralogs (Poppenberger et al. 2006) (Fig. 1A). Also, the genomes of rice and the model grass species Brachypodium distachyon (Opanowicz et al. 2008) contain clusters of highly similar UDP-glycosyltransferase (UGT) genes, most of which are expected to code for enzymes transferring glucose to small molecules. The enormous size of the small molecule conjugating the UGT gene family (with approximately 100 to 140 members in different diploid plant species) (Bowles et al. 2006) makes it difficult to attribute a particular enzymatic activity to a particular gene. Numerous candidate UGT genes with a possible role in DON detoxification have been identified in wheat and barley, based on evidence such as upregulation in response to Fusarium spp. infection, induction upon DON treatment, or differential expression in cultivars differing in Fusarium disease resistance (Hill-Ambroz et al. 2006; Desmond et al. 2008; Walter et al. 2008; Steiner et al. 2009). Using the Affymetrix Barley1 GeneChip, nine UGT probe sets upregulated during Fusarium spp. infection had been identified (Boddu et al. 2006). A subset of three UGT was specifically induced upon infection with wild-type $F$. graminearum but not if a tri5 mutant deficient in DON production was used as inoculum (Boddu et al. 2007). In a complementary study of barley treated with DON, two UGT genes were found exhibiting more than 15- and 90-fold increases in transcript accumulation compared with water treatment (Gardiner et al. 2010). Recently, six DON-induced wheat UGT were identified utilizing the Affymetrix wheat GeneChip (Lulin et al. 2010), and the authors attempted to validate the proposed function of one candidate by overexpression in Arabidopsis (Lulin et al. 2010). However, the phenotypic difference in DON resistance between the Arabidopsis wild type and the transformed line is much weaker than reported for overexpression of UGT73C5 (Poppenberger et al. 2003). In this study, we utilized heterologous expression of candidate UGT in yeast to test whether the Arabidopsis genes forming a cluster with UGT73C5 have the same substrate specificity and protective activity against DON. The results show that it is difficult to predict substrate specificity of monocotyledonous UGT based on sequence similarity. Therefore, we experimentally tested the most promising barley candidates identified in the accompanying article (Gardiner et al. 2010). Furthermore, we reinvestigated the functionality of a gene from wheat (TaUGT3) for which DON-glucosyltransferase activity was claimed (Lulin et al. 2010). Our results indicate that only one of the four barley candidates functioned as a DON-glucosyltransferase leading to DON resistance in yeast, and that the wheat TaUGT3 gene is inactive. Therefore, validation of the proposed function of a candidate UGT gene is highly recommended before investing resources into breeding efforts or transgenic approaches aiming to improve DON resistance of crop plants.

\section{RESULTS}

DON detoxification

by Arabidopsis UGT73C cluster members.

UGT73C5 (DOGT1, At2g36800) from Arabidopsis encodes an enzyme that can inactivate DON by forming the glucose conjugate D3G (Poppenberger et al. 2003). UGT73C5 belongs to the subfamily $73 \mathrm{C}$ of UGT of $A$. thaliana (Li et al. 2001) and is clustered with other members of the subfamily (UGT73C1-C6) in a tandem repeat. The six genes do not con- 
tain introns and show very high similarity to each to each other in the coding regions. UGT73C6 located next to UGT73C5 encodes a protein with $89.7 \%$ amino-acid identity (Table 1 ).

The high similarity of $U G T 73 C 1, C 2, C 3, C 4, C 5$ (=DOGT1), and $C 6$ and their clustering (Fig. 1A) suggests that they have evolved by gene duplications from an ancestral gene and, therefore, might still have similar enzymatic properties. To test this hypothesis, the reading frames of the cluster members were cloned into a yeast expression vector with an $\mathrm{N}$-terminal $c$ Myc epitope tag, as previously described (Poppenberger et al. 2006). The resulting plasmids were introduced into the DONsensitive yeast strain YZGA515 (Table 2). Expression of the expected UGT gene products was confirmed by Western blotting as previously described (Poppenberger et al. 2006). Spotting of suspensions of the transformed yeast to YPD (yeast peptone dextrose) plates containing increasing amounts of DON revealed surprising differences in the ability to protect against DON. In addition to $U G T 73 C 5$, the originally identified gene (Poppenberger et al. 2003), UGT73C4 conferred a high level of DON resistance (e.g., DON at $120 \mathrm{mg} / \mathrm{liter}$ and higher) (Fig. 1B). In contrast, UGT73C6-which has the highest similarity with $U G T 73 C 5$-was only able to confer marginal resistance. The difference in resistance between UGT73C6 and the empty vector control could only be observed in a very narrow concentration range $(\leq 30 \mathrm{mg} / \mathrm{liter})$. The $U G T 73 C 3$ gene product, which is most similar (76.1\% identical) to UGT73C4, showed a comparably low DON detoxification ability, similar to UGT73C6 in the plate assays. The different characteristics of the closely related Arabidopsis gene products opened the possibility to elucidate features of the proteins which are crucial for DON recognition. An EcoRI site present in both UGT73C5 and UGT73C6 was used to construct hybrid genes containing the N-terminal part of one and the C-terminal 307 amino acids of the other gene. Expression in yeast and exposure to DON revealed that the N-terminus of UGT73C5 (consisting of the first 189 amino acids) is essential for the ability to detoxify DON. However, the resistance level conferred by the hybrid protein C5-C6 (Fig. 1C) is much lower than wildtype C5. We next looked for sequence differences in the suspected substrate-binding region (Vogt et al. 1999), a hydrophobic area between amino acids 130 and 150 . The lysine residue 136 in UGT73C5 was chosen for site-directed mutagenesis. It is conserved in the sequence of the other detoxifying UGT73C4 but replaced by other amino acids in the two nondetoxifying paralogs UGT73C3 and UGT73C6. We changed $\mathrm{K} 136$ in UGT73C5 (DOGT1) to glutamic acid, which is present at the corresponding position (amino acid 137) in UGT73C6. The mutant UGT73C $5^{\mathrm{K} 136 \mathrm{E}}$ protein protected with the same efficiency as wild-type UGT73C5 (data not shown). Also, the corresponding construct UGT73C $6^{\mathrm{E} 137 \mathrm{~K}}$ remained inactive, thus demonstrating that K136 is not essential for the DON detoxification ability.

Previously, we showed that the UGT73C5 gene encoding the DON protective enzyme is highly induced by DON at $5 \mathrm{mg} / \mathrm{liter}$ in Arabidopsis (Poppenberger et al. 2003). In this study, we tested this for the genes UGT73C4 and UGT73C6. Reversetranscriptase polymerase chain reaction (PCR) revealed that

Table 1. Percent amino-acid identity of products of the Arabidopsis thaliana UGT73C gene cluster

\begin{tabular}{lccccc}
\hline Gene & $\boldsymbol{C 2}$ & $\boldsymbol{C 3}$ & $\boldsymbol{C 4}$ & $\boldsymbol{C 5}$ & $\boldsymbol{C 6}$ \\
\hline C1 & 80.5 & 78.3 & 72.2 & 74.4 & 73.6 \\
C2 & $\ldots$ & 86.5 & 75.2 & 78.0 & 77.6 \\
C3 & $\ldots$ & $\ldots$ & 74.2 & 77.0 & 76.4 \\
C4 & $\ldots$ & $\ldots$ & $\ldots$ & 74.2 & 73.4 \\
C5 & $\ldots$ & $\ldots$ & $\ldots$ & $\ldots$ & 86.9 \\
\hline
\end{tabular}

the other two genes are DON inducible (Fig. 1D), regardless of whether or not their products provide protection against DON. The inactive gene UGT73C6 showed an even stronger induction of expression by DON than UGT73C5 or UGT73C4. Transcript accumulation was observed already after $1 \mathrm{~h}$ of incubation with the toxin, reaching a peak after $4 \mathrm{~h}$ and declining again between 6 and $12 \mathrm{~h}$ (Fig. 1D).

Overall, we conclude from our Arabidopsis results that it is not possible to predict substrate specificity based on sequence similarity even in the case of very closely related genes. Also, inducibility does not seem to be a good predictor of function. Therefore, it seems unlikely that genes from monocotyledonous crop plants with much lower similarity will generally have the presumed substrate specificity. At least, it is highly advisable to experimentally test such hypotheses and to validate the function of candidate genes.

\section{Identification of a barley UGT with DON-detoxification capability.}

In an accompanying study, the fate of DON in barley was investigated and candidate UGT genes potentially responsible for formation of D3G were identified using the Barley1 Affymetrix GeneChip (Gardiner et al. 2010). DON injected into barley spikelets disappeared faster if only a single spikelet and not the whole ear was treated with concentrations of DON that are expected to completely inhibit protein biosynthesis (200 $\mathrm{mg} / \mathrm{liter}$ ). A possible explanation for this observation is that DON is diluted by transport to a concentration level that allows translation of a DON-induced UGT gene whereas, if the whole tissue is treated, only little constitutive activity is available for DON detoxification. By comparing gene expression in DON- and water-treated barley, barley infected with a DON-producing Fusarium strain and a tri5 mutant, and mockinfected barley, four transcripts corresponding to candidate UGT genes were selected for further analysis: Hv14077, Hv13248, Hv19290, and Hv5876.

The full-length cDNA clones of HvUGT5876, HvUGT14077, HvUGT19290, and HvUGT13248 were cloned into pGEM vectors (discussed below). All four barley genes (submitted gene bank accession numbers in parentheses) have predicted reading frames of typical length: HvUGT5876 (GU170354), 1,491 bp; HvUGT14077 (GU170356), 1,473 bp; HvUGT19290 (GU170357), 1,461 bp; and HvUGT13248 (GU170355), 1,428 bp. Sequence comparison revealed little

Table 2. Yeast strains used in this study

\begin{tabular}{|c|c|c|}
\hline $\begin{array}{l}\text { Strain } \\
\text { name }\end{array}$ & $\begin{array}{l}\text { Plasmid } \\
\text { introduced }\end{array}$ & $\begin{array}{c}\text { Relevant genotype/alteration relative to } \\
\text { parental strain YZGA515 }\end{array}$ \\
\hline YZGA515 & $\ldots$ & $\begin{array}{l}\text { MATa leu2-41 trp1- } 463 \text { ura3-52 his3-4200 } \\
\text { lys2-801 ade2-101 pdr5::TRP1 pdr10::hisG } \\
\text { pdr15:loxP-KAN -loxP ayt1::URA }\end{array}$ \\
\hline YZBP51 & pBP868 & $2 \mu-L E U 2-P_{A D H I}-c-M Y C-A t U G T 37 C 5$ \\
\hline YZBP55 & pBP874 & $2 \mu-L E U 2-P_{A D H I}-c-M Y C-A t U G T 37 C 1$ \\
\hline YZBP57 & pBP1378 & $2 \mu-L E U 2-P_{A D H 1}-c-M Y C-A t U G T 37 C 2$ \\
\hline YZBP59 & pBP901 & $2 \mu-L E U 2-P_{A D H I}-c-M Y C-A t U G T 37 C 3$ \\
\hline YZBP61 & pBP1374 & $2 \mu-L E U 2-P_{A D H 1}-c-M Y C-A t U G T 37 C 4$ \\
\hline YZBP63 & pBP918 & $2 \mu-L E U 2-P_{A D H 1}-c-M Y C-A t U G T 37 C 6$ \\
\hline YZBP65 & pBP910 & $2 \mu-L E U 2-P_{A D H I}$ (empty vector) \\
\hline YZBP81 & pBP1342 & $2 \mu-L E U 2-P_{A D H I}-c-M Y C-A t U G T 37 C 6 / C 5$ \\
\hline YZBP83 & pBP1346 & $2 \mu-L E U 2-P_{A D H I}-c-M Y C-A t U G T 37 C 5 / C 6$ \\
\hline YZBP91 & pBP1663 & $2 \mu-L E U 2-P_{A D H I}-c-M Y C-A t U G T 37 C 5-K 136 E$ \\
\hline YZBP93 & pBP1669 & $2 \mu-L E U 2-P_{A D H I}-c-M Y C-A t U G T 37 C 6-E 137 K$ \\
\hline YZWS124 & pWS1925 & $2 \mu-L E U 2-P_{A D H 1}-c-M Y C-H v U G T 19290$ \\
\hline YZWS125 & pWS1921 & $2 \mu-L E U 2-P_{A D H I^{-}}-\mathrm{MYC}-H \nu U G T 13248$ \\
\hline YZWS126 & pCE2217 & $2 \mu-L E U 2-P_{A D H 1}-c-M Y C-H v U G T 14077$ \\
\hline YZWS127 & pCE2228 & $2 \mu-L E U 2-P_{A D H I}-c-M Y C-H \nu U G T 5876$ \\
\hline YZWS164 & pWS2167 & $2 \mu-L E U 2-P_{A D H 1}-c-M Y C-T a U G T 3$ \\
\hline YZDL112 & $\ldots$ & YZGA515 except $p d r 5::$ loxP \\
\hline YZBP3 & $\ldots$ & YZGA515 except ugt51::loxP-SpHIS5-loxP \\
\hline
\end{tabular}


amino-acid similarity between the products of the barley genes and AtUGT73C5. The maximum amino-acid identity within the group of Hordeum UGT was only 35\% (HvUGT5876 and HvUGT19290). A higher sequence identity could be found when comparing HvUGT5876 with AtUGT73C5 (43\%). HvUGT13248 showed the lowest identity (23\%) with AtUGT73C5.

The basic strategy for testing substrate specificity by heterologous expression in yeast was to clone the UGT inserts in frame downstream of the $\mathrm{N}$-terminal c-Myc tag. The yeast expression vectors for the HvUGT genes were constructed by homologous recombination in yeast. First, the sequences corresponding to the short $\mathrm{N}$ - and $\mathrm{C}$-terminal fragments separated by a unique site for linearization and flanked by HindIII and NotI sites were custom synthesized. The sequences and resulting plasmids are given in Supplementary Table 1. The HindIIINotI fragments were moved into the yeast expression vector pYAK7, giving the gap repair vectors indicated in Table 3 . The linearized gap repair vectors were used together with the corresponding cDNAs to transform yeast. A schematic representation of the homologous recombination procedure is shown in Figure 2.

The resulting UGT expression vectors (Table 2), a negative control (pBP910, empty vector), and a positive control (pBP868, AtUGT73C5) were transformed into the toxin-sensitive yeast strain YZGA515. After 2 days on toxin medium, the transformants containing the barley UGT13248 expression vector showed growth (Fig. 3), also at concentrations (above $200 \mathrm{mg} / \mathrm{liter}$ ), that were inhibitory for the positive control AtUGT73C5 (data not shown). In contrast, transformants con-

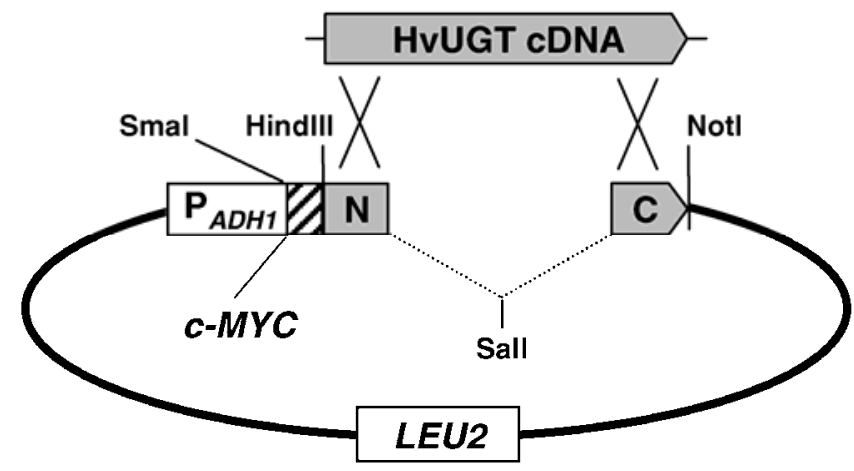

Fig. 2. Construction of expression vectors by homologous recombination in yeast. Linearized gap repair vectors containing short $\mathrm{N}$-terminal $(\mathrm{N})$ and C-terminal (C) fragments of a given barley glucosyltransferase cDNA (HvUGT) were co-transformed with the respective cDNA inserts. In the resulting products of in vivo recombination $(\mathrm{X})$, the open reading frames of the cDNAs with an N-terminal $c-M Y C$-epitope tag are placed downstream of the yeast $A D H 1$ promoter. taining the vectors with the HvUGT 5876, 14077, or 19290 constructs showed the same toxin sensitivity as the control strain with the empty vector. One possible reason for the failure to protect could be that the respective proteins are not expressed or expressed only at very low levels. Using Western blotting with the antibody detecting the c-Myc-tag, we confirmed that the other proteins not conferring resistance against DON are clearly expressed, although not at the same high level as HvUGT13248 (Fig. 3).

\section{Reinvestigating the activity}

of the candidate wheat gene TaUGT3.

While this work was in progress, the cloning and characterization of the supposedly first crop gene designated "DON-resistance related TaUGT3" was reported (Lulin et al. 2010). This claim is based on an Arabidopsis transformant which showed less inhibition by DON than the untransformed control line. However, the phenotypic difference shown (increased root length and leaf area) is far less pronounced than previously demonstrated for UGT73C5 overexpression (green seedlings of transformants compared with bleached wild-type seedlings showing growth arrest without formation of true leaves) (Poppenberger et al. 2003). Furthermore, no direct evidence for D3G formation by TaUGT3 was presented. The amino-acid sequence of TaUGT3 (GenBank accession number FJ236328) is highly similar (88.9\%) to the barley HvUGT5876, which was inactive in the yeast assay. We cloned the TaUGT3 cDNA

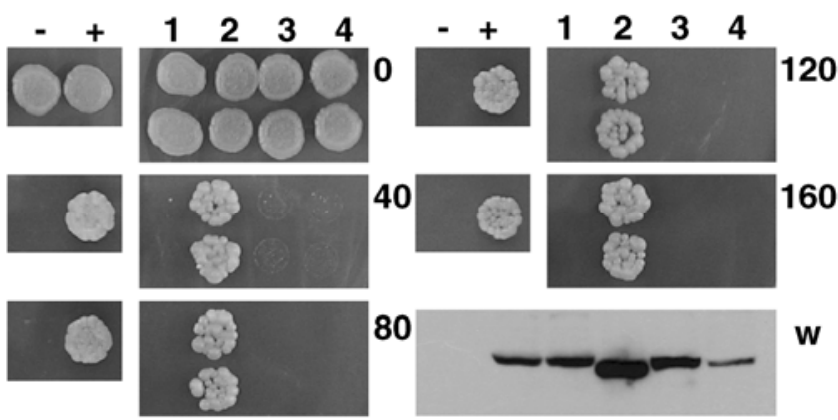

Fig. 3. Deoxynivalenol (DON)-resistance phenotype conferred by expression of candidate barley UDP-glycosyltransferases (UGT) in yeast. Transformants of strain YZGA515 containing the negative control $(-=$ empty vector, $\mathrm{pBP} 910)$ and positive control $(+=A t U G T 73 C 5$ expression vector, pBP868) and the candidate barley UGT were spotted on plates containing the indicated concentration of DON (mg/liter). The control plasmids are shown in the small boxes on the left. Two independent transformants with the $H v U G T$ expression vectors were spotted (one in upper row and one in lower row). The strains received the following numbers. 1, HvUGT5876; 2, HvUGT13248; 3, HvUGT14077; and 4, HvUGT19290. This strain code was also used in the Western blotting experiment shown in the lower right column $(-=$ empty vector and $+=A t U G T 73 C 5)$.

Table 3. Plasmids generated during construction of the HvUGT expression vectors

\begin{tabular}{lccccc}
\hline Gene designation (accession no.) & $\begin{array}{c}\text { Adapter fragment } \\
\text { plasmid }^{\mathbf{b}}\end{array}$ & $\begin{array}{c}\text { Gap repair } \\
\text { plasmid }^{\mathbf{c}}\end{array}$ & $\begin{array}{c}\text { UGT full-length } \\
\text { cDNA plasmid }^{\mathbf{d}}\end{array}$ & $\begin{array}{c}\text { cDNA fragment } \\
\text { release by }^{\mathbf{e}}\end{array}$ & $\begin{array}{c}\text { Resulting expression } \\
\text { vector }^{\mathbf{e}}\end{array}$ \\
\hline HvUGT5876 (GU170354) & pWS1891 & pWS1906 & pCE2158 & NotI & pCE2228 \\
HvUGT13248 (GU170355) & pWS1893 & pWS1911 & pCE2149 & SphI + SpeI & pWS1921 \\
HvUGT14077 (GU170356) & pWS1889 & pWS1898 & pCE2152 & EcoRI & pCE2217 \\
HvUGT19290 (GU170357) & pWS1895 & pWS1920 & pCE2155 & SphI + SpeI & pWS1925 \\
\hline
\end{tabular}

${ }^{a}$ GenBank accession numbers for the full-length cDNAs.

${ }^{\mathrm{b}}$ Synthetic sequences corresponding to the $\mathrm{N}$ - and C-terminal fragments were cloned into the adapter fragment plasmids.

${ }^{\mathrm{c}}$ Inserts were HindIII-NotI released and transferred into pYAK7, yielding the indicated gap repair plasmids. These were linearized with SalI (or BglII in case of HvUGT13248) for yeast transformation.

${ }^{\mathrm{d}}$ UGT $=$ UDP-glycosyltransferase.

${ }^{\mathrm{e}}$ HvUGT cDNA fragments were released by digestion with the enzymes shown from the full-length cDNA plasmids (UGT in vector pGEMT-Easy) and cotransformed with the linearized gapped vector to create the resulting yeast expression vectors by homologous recombination in vivo. Expression vectors were recovered, sequenced, and retransformed into yeast strain YZGA515. 
into our expression vector for testing. Spotting yeast transformants on DON plates revealed that TaUGT3 did not confer resistance (Fig. 4), like the putative barley ortholog HvUGT5876. To exclude that this is caused by insufficient expression, we also tested expression by Western blotting. The TaUGT3 was expressed in yeast at a level similar to the positive control AtUGT73C5 (Fig. 4). Semiquantification of the Western blot by densitometry confirmed this observation.

\section{Confirmation of D3G formation by HvUGT13248.}

The yeast strain containing the expression vector with the $H v U G T 13248$ insert was used to confirm that the resistance phenotype is due to formation of D3G. DON was added stepwise to a final concentration of $500 \mathrm{mg} /$ liter to cultures growing on glucose medium. Remaining DON and D3G was determined by high-performance liquid chromatography tandem mass spectrometry (HPLC-MS/MS) as previously described (Poppenberger et al. 2003; Berthiller et al. 2009a). To avoid a difference in growth between the DON-sensitive vector control and the resistant UGT transformants (HvUGT13248 and AtUGT73C5 positive control), we transformed the strains for this experiment with a plasmid containing a gene encoding a toxin-resistant form of ribosomal protein L3, as previously described (Poppenberger et al. 2003, Mitterbauer et al. 2004). In a control experiment without the $R P L 3^{W 255 C}$ plasmid (pRM561), the yeasts carrying the HvUGT13248 expression vector also showed high D3G production (more than 500 $\mu \mathrm{g} /$ liter). The strain with the Arabidopsis UGT73C5-positive control plasmid pBP868 produced D3G at approximately 100 $\mu \mathrm{g} /$ liter without and $150 \mu \mathrm{g} /$ liter in the presence of pRM561. In YZGA515 cells expressing the HvUGT13248 without the $R P L 3^{W 255 C}$ plasmid after $24 \mathrm{~h}, \mathrm{D} 3 \mathrm{G}$ at up to $747 \mu \mathrm{g} /$ liter was present in the supernatant, and calculated $270 \mu \mathrm{g}$ of cell-associated D3G per liter of culture. The time course of D3G present in cells and culture supernatant of transformants expressing the HvUGT13248 is shown in Figure 5.

\section{DISCUSSION}

Detoxification reactions catalyzed by glucosyltransferase seem to play a far more prominent role in plant pathogen interactions than currently assumed. For instance, detoxification of the Alternaria brassicae virulence factor destruxin B by glucosidation is the rate-limiting step in the resistant Brassica spp. (Pedras et al. 2002). Vice versa, detoxification of the plant defense compound brassinin by a fungal pathogen (Sclerotinia) UGT was recently demonstrated (Sexton et al. 2009). Glucosyltransferases may have specific preferences for different activated sugar co-substrates. In plants, most UGT seem to prefer UDP-glucose. In herbivorous animals, the large gene

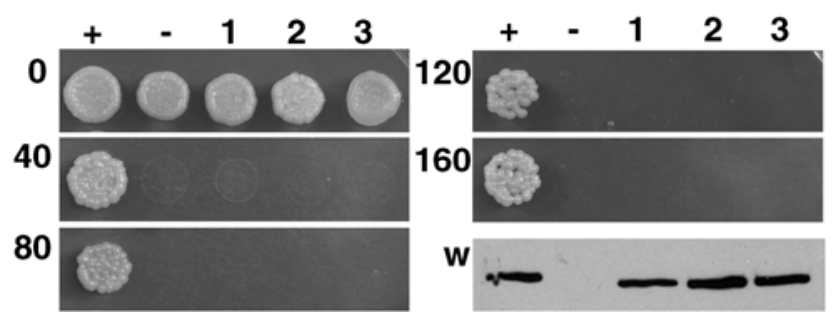

Fig. 4. Expression of TaUGT3 in yeast does not confer deoxynivalenol (DON) resistance. Growth of yeast strains containing a positive control plasmid ( $+=A t U G T 73 C 5$ expression vector, pBP868), a negative control ( $-=$ empty vector, $\mathrm{pBP} 910)$, and three independent transformants expressing TaUGT3 (1-3) on YPD plates containing the indicated concentration of DON (mg/liter). Panel in the lower right column shows the respective Western blot (w) with the c-Myc antibody. family of UDP-glucuronosyltransferases is highly relevant for detoxification of plant metabolites (Bock 2003). In the case of the mammalian UGT1A gene family, between 10 and 14 alternative first exons, which encode the $\mathrm{N}$ termini responsible for different substrate specificity, are tandemly arrayed upstream of four shared exons coding for the common $C$ terminus. In contrast, multiple independent loci for different $U G T 2 B$ glucuronosyltransferase genes and pseudogenes are clustered in a chromosomal region of humans (Owens et al. 2005). Also, in plants, whole genes seem to by amplified, possibly during toxin stress. Subsequent diversifying selection or genetic drift could play a role in evolution of UGT gene clusters. By constructing hybrid genes between the closely related AtUGT73C5 and $C 6$, we found that the N-terminal part of UGT is responsible for substrate specificity (Fig. 1C), which is in agreement with the proposed two-domain structure (Osmani et al. 2009). Recently, Hansen and associates (2009) reported that 12 of 20 chimeric genes constructed were enzymatically active. Such chimeras can have pronounced differences in substrate specificity and regioselectivity, leading to different products having glucose or other sugars attached to different (e.g., hydroxyl) groups in a molecule. Hypothetically, recombination between genes in a cluster or between other, more distant UGT in the genome could play an important role in the evolution of new UGT specificities by providing protection against microbial toxins.

The results of the characterization of members of the Arabidopsis gene cluster and the barley candidate UGT genes suggested that sequence similarity is not a meaningful predictor of substrate specificity. This is in line with previous reports showing similar substrate specificity for unrelated UGT and contrasting activity in gene products with high sequence similarity. For instance, a gene from Arabidopsis, with similarity to an indole-3-acetic acid (IAA) conjugate forming UGT from maize, was initially annotated as IAA-glucosyltransferase. However, the encoded protein was found inactive in vitro with auxin. In contrast, AtUGT84B1, which is completely unrelated to the maize IAA-UGT, had very high activity with auxin, whereas the highly similar AtUGT84B2 had only trace activity (Jackson et al. 2001). Contrasting activity was also observed by us for the UGT73C cluster members with respect to DON detoxification. Although UGT73C5 and C4 conferred resis-

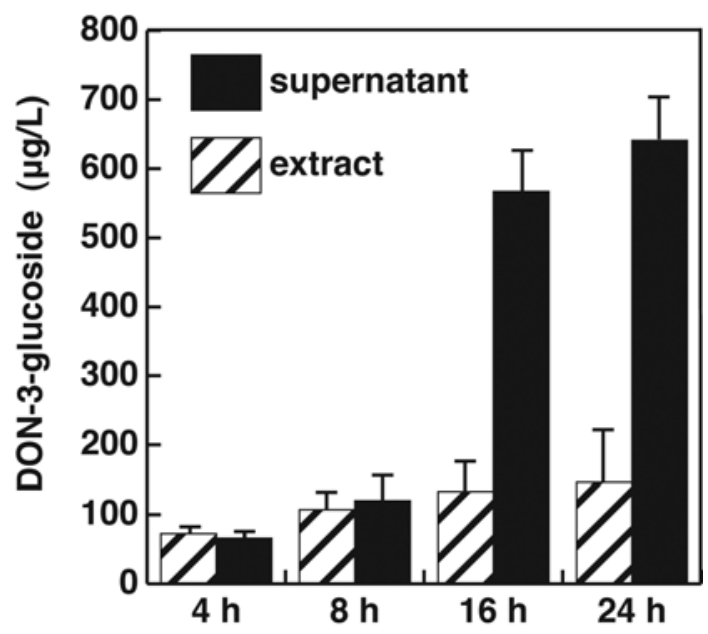

Fig. 5. Conversion of deoxynivalenol (DON) into DON-3-O-glucoside (D3G) and release into the culture fluid by transformed yeast. Time course of D3G (micrograms of D3G per liter of culture) in the medium and associated with cells. Shown are the mean values and standard deviations of seven independent transformants expressing HvUGT13248 at the indicated time points. 
tance in yeast, UGT73C6 did not. The UGT73C6 gene product was previously shown to have very high affinity and activity against the Fusarium toxin zearalenone (Poppenberger et al. 2006). Also, the UGT73C1 and UGT73C2 constructs did not confer toxin resistance in yeast, which could be partly due to lower expression levels of these proteins (Poppenberger et al. 2006). Activity toward different hydroxycoumarins has been shown for all 73C cluster members (Lim et al. 2003), indicating that the UGT73C genes lacking DON-protective activity in our study are not simply loss-of-function alleles. It is well documented for mammalian UDP-glucuronosyltransferases that single amino-acid changes can have profound effects on detoxification activity (Guillemette 2003).

The mere size of the small-molecule-conjugating glucosyltransferase gene family (with an estimated 140 UGT genes in the sequenced barley relative, genus Brachypodium) makes it very difficult to identify genes with specific enzymatic activity, such as DON-glucosyltransferase. The approach to narrow down candidates in this study was based on the finding that detoxification is inducible. Results of genome-wide expression analysis allowed research to focus on four candidate genes (Gardiner et al. 2010). However, the Arabidopsis results also indicate that genes lacking detoxification activity can be highly inducible (Fig. 1D), and only one (HvUGT13248) out of the four tested DON-induced barley UGT genes conferred resistance against DON. We cannot exclude that other untested DON-induced or constitutively expressed UGT have DON-detoxification properties. In principle, the protective activity is a function of both substrate specificity and gene expression level. It is also obvious that more than 100 UGT genes cannot all be highly expressed at the same time. Therefore, nucleotide differences in promoter regions, which affect basal level or induction kinetics, may also be responsible for differences in DON resistance between cultivars.

The barley gene product HvUGT5876 which showed the highest similarity to UGT73C5 turned out to be inactive against DON, whereas the gene product with the lowest identity (23\%) with AtUGT73C5, HvUGT13248, had the desired specificity of a DON-glucosyltransferase. Despite enormous progress in molecular modeling of UGT (Osmani et al. 2009) based on solved crystal structures, the prediction of substrate specificity from the amino-acid sequence is still imperfect. Sequence comparison of two Arabidopsis UGT with protective effect ( $73 C 4$ and $C 5)$ and two genes ( $73 C 3$ and $C 6)$ with very low activity led to the hypothesis that a lysine at position 136 in UGT74C5 but replaced in the two UGT unable to detoxify DON could be important for specificity. However, this simple concept was falsified using the yeast assay.

The yeast heterologous expression system and determination of the resistance phenotype is a straightforward and simple strategy for experimental testing of glucosyltransferases compared with purification of the recombinant protein and characterization by in vitro assays. A prerequisite is the use of an engineered yeast strain with a low level of DON resistance due to reduced drug efflux (inactivated ABC transporter genes PDR5 PDR10 PDR15) and inactivation of the $A Y T 1$ gene (coding for an acetyltransferase that converts DON into 3-acetyl-DON) (Alexander et al. 2002). Yeast is devoid of small-molecule UGT, with the exception of a sterol glucosyltransferase (ATG26/UGT51/YLR189C) which does not play a role in DON resistance in yeast (data not shown) (Poppenberger 2003). Therefore, addition of a plant candidate gene allows undisturbed observation of the phenotype caused by the heterologous protein. A potential problem, not encountered by us thus far, is that certain proteins may be recalcitrant to expression in yeast or that mRNAs are processed incorrectly (e.g., leading to premature termination and polyadenylation or wrong splicing). Therefore, verification that the protein is expressed is necessary. A potential limitation of yeast is that it does not contain certain activated donor sugars such as UDP-rhamnose, UDP-xylose, or UDPglucuronic acid, so that rhamnosyltransferases, xylosyltransferases, or glucuronosyltransferases are expected to be inactive in yeast due to lack of the co-substrate. No evidence for formation of such DON conjugates in plants is currently available. Glucose conjugate formation seems to be the predominant mechanism of glycosylation of DON. Theoretically, the overexpression in Arabidopsis or other plants may allow detection of glucosyltransferases active with sugar donors other than glucose. Arabidopsis, on the other hand, has the disadvantage of multiple (inducible) endogenous genes that most likely confound the desired phenotype. In principle, we cannot exclude the possibility that the weak resistance phenotype reported for transgenic Arabidopsis overexpressing TaUGT3 is due to a glycosylation reaction that is not occurring in yeast. However, it seems more likely that an Arabidopsis somaclonal variant with slightly better resistance was chosen for analysis by Lulin and associates (2010) and that the TaUGT3 gene, which is inactive in yeast, is also irrelevant for DON detoxification in wheat.

The biggest challenge in identifying the desired glucosyltransferases from crop plants remains the cloning of fulllength cDNAs when full genome sequences are not available. Microarray and expressed sequence tag (EST) data typically allow the identification of the highly conserved C-terminal UGT sequences responsible for binding the activated sugars (PSPG box, binding of UDP-glucose). To link up the corresponding $\mathrm{N}$ terminus responsible for substrate specificity in the presence of numerous confounding genes is not trivial. Especially problematic is hexaploid wheat, where not only other cluster members with highly similar sequences but also nearly identical homologous sequences from the other ancestral genomes exist. In wheat, cases of unintentional "sexual PCR" (where nearly identical fragments of cDNAs from different genes are recombined) (Stemmer 1994) may easily occur and complicate the issue (W. Schweiger, unpublished).

Also, the barley sequences we obtained by rapid amplification of cDNA ends (RACE) PCR do not line up perfectly with available EST sequences (Supplementary Table 2). This could be due to sequencing errors in EST, cultivar-specific allelic differences, or, potentially, PCR errors during the cDNA cloning. The result of resequencing of Morex cDNAs revealed that our full-length cDNA HvUGT13248 contains a mutation (A843C) leading to a change of proline to leucine. This amino-acid alteration obviously does not interfere with the function of the gene product as a DON-glucosyltransferase. The other cDNAs were confirmed (HvUGT14077 and HvUGT19290) or only contained a silent change (HvUGT5876).

Once full-length sequences are available, the described yeast homologous recombination approach allows rapid creation of expression vectors without further PCR or difficult cloning steps. The crystal structure of a Medicago UGT (Shao et al. 2005) shows that the $\mathrm{N}$ terminus is flexible, so that addition of the epitope tag should not interfere with enzymatic function. The different barley UGT did show variations in expression level but the differences observed are too low to explain the complete lack of protective activity of some genes. Because the task is not to validate the absence of activity but to find the rare gene with the desired activity, the yeast system provides a suitable rapid and qualitative test. For a quantitative biochemical comparison, enzyme purification and characterization of the catalytic properties in vitro would be needed. The tagged protein from yeast could also be used directly for purification with c-Myc affinity columns. 
The use of living Escherichia coli cells expressing UGT as biocatalysts for formation of glucosides has been described (Lim 2005) but was unsuccessful in our case with UGT73C5 and DON (data not shown). Toxin conjugates accumulated in the medium of yeast cells transformed with AtUGT73C5 and HvUGT13248 upon treatment with DON (Fig. 5). We have successfully employed the engineered yeast strain expressing AtUGT73C6 for production of zearalenone- and zearalenolglucosides (Berthiller et al. 2009b). In the case of D3G, the yield obtained with yeast is still very low compared with other substances. At present, we do not understand why certain glucose conjugates are more efficiently exported than others, and which yeast genes are required for this process. The general assumption that glucosides accumulate only in the vacuole may be incorrect in plants. Of the total D3G formed after $24 \mathrm{~h}$ by yeast, only $19 \%$ was found within the cells; the rest was found in the medium (Fig. 5). Excretion of glucosides (and subsequent incorporation into plant cell wall material) instead of actively concentrating the conjugate in the vacuole could be more important than currently anticipated. Practically nothing is known about the bioavailability of DON in the insoluble residue (covalently bound to lignin and cell wall components), whereas the "masked mycotoxin" issue - the possible hydrolysis of soluble D3G back to DON in the intestines of humans and animals - increasingly receives attention. In general, resistance breeding to counteract the virulence factor DON seems valid, because plants with higher DON resistance can better restrict fungal spread, leading to lower amounts of fungal biomass and total DON + D3G. However, clearly, other Fusarium virulence factors besides DON exist, and cultivars with still poor Fusarium disease resistance but high DON-detoxification capability may contain higher molar ratios of D3G/DON than found now.

For Fusarium disease resistance breeding purposes and biotechnological approaches, a validation of the proposed substrate specificity of candidate UGT genes is highly warranted, to avoid wasting time and resources on irrelevant genes. Using the yeast expression system, the proposed function of TaUGT3 as a DON-glucosyltransferase could be rapidly falsified. The identification of the first monocot DON-glucosyltransferase gene, $H v U G T 13248$, should provide an important entry point to transfer this knowledge to other crop species and, hopefully, will lead to a rapid progress in Fusarium disease resistance breeding and mycotoxin reduction efforts.

\section{MATERIALS AND METHODS}

\section{Plasmid constructs and yeast strains} expressing Arabidopsis UGT.

The construction of the expression vectors for the AtUGT73C cluster and the yeast expression vector pYAK7 was described previously (Poppenberger et al. 2006). For the domain-shuffling experiment, the open reading frames (ORF), including the N-terminal $c-M y c$ tag of DOGT1 and its closest homologue, UGT73C6, were isolated from the yeast expression vectors by SmaI + NotI digestion (Fig. 2), cloned into vector pBluescript SKII+ (which was cut with XhoI, treated with Klenow enzyme, and subsequently digested with NotI). The resulting plasmids were digested with HindIII and a conserved EcoRI site present in both genes that cleaves DOGTI at nucleotide position 565 (73C6 at 568). Hybrids were constructed by ligation of the $\mathrm{N}$-terminal part of one gene to the C-terminal part of the other. The resulting hybrid fragments were moved back into the yeast expression vector pYAK7 using the HindIII and NotI restriction sites, and the resulting plasmids (Table 3) were transformed into YZGA515 (Table 2). Mutations were constructed by overlap extension PCR using overlapping mutant primers DOG-K136E-fw (5'-TACAAGCG AAATCGCCAAGAAGTTCA-3') and DOG-K136E-rv (5'-CT TCTTGGCGATTTCGCTTGTATAAG-3') and flanking primers DOGIpYAK7-fw-a (5'-ACTAAGCTTGGAATCATGGTTTCC GAAACA-3') and DOG-EcoRI-rv (5'-TCTTGTGAATTCAA CTCTATCAGGA-3') for mutagenesis of DOGT1 (73C5).

Mutant primers 73C6-E137K-fw (5'-TACAAGCAAAATC GCCAAGAAGTTCAA-3') and 73C6-E137K-rv (5'-ACTTCT TGGCGATTTTGCTTGTATT-3') and flanking primers 73C6pYAK7-fw (5'-CTAAGCTTGGAATCATGTGTTCTCATGAT CCT-3') and DOG-EcoRI-rv were used for $73 C 6$ mutagenesis. The resulting PCR products were cloned as HindIII + EcoRI fragments into the corresponding genes present in vector pBluescript SKII+. After sequencing, the ORF were moved as HindIII + NotI fragments back into the yeast expression vectors and the resulting plasmids were transformed into YZGA515 (Table 2).

\section{Analysis of DON-induced gene expression in Arabidopsis.}

Arabidopsis seedlings grown in liquid Murashige-Skoog medium were treated with DON at $5 \mathrm{mg} /$ liter and total RNA was isolated. The cDNA was synthesized from $1 \mu \mathrm{g}$ of total RNA (digested with DNaseI) with $500 \mathrm{ng}$ of a 18-mer oligo-dT and reverse transcriptase. PCR was performed with approximately $2 \mu \mathrm{l}$ of the 1:20 diluted cDNA using primers that amplify 200- to 400-bp large fragments located in the C-terminal part of the genes to be analyzed (DOGRT-fw: 5'-ATCCGGG GTTGAACAGCCT-3', DOGRT-rv: 5'-TCAATTATTGGGTT CTGCC-3'; 73C4RT-fw: 5'-GGAGAAAATAGGAGTGTTA-3', 73C4RT-rv: 5'-TCAGTTCTTGGATTTCACT-3'; 73C6RT-fw: 5'-GAGAAACTGGTCGTACAA-3', and 73C6RT-rv: 5'-TCA ATTATTGGACTGTGCT-3'). As a control for normalization, primers corresponding to $U B Q 5$ (coding for an ubiquitinRPS27 fusion protein) were used: UBQ5-U, 5'-GTCCTTCTT TCTGGTAAACGT-3', and UBQ5-D, 5'-AACCCTTGAGGTT GAATCATC-3'.

\section{Cloning of barley full-length cDNAs and construction of yeast expression vectors.}

Based on the sequence information available for Contig5876_at (HvUGT5876), Contig14077_at (HvUGT14077), Contig19290_at (HvUGT19290), and Contig13248_at (HvUGT13248) in the harvEST database, several strategies were employed to clone full-length cDNA sequences.

For HvUGT5876, a full-length cDNA could be deduced from the available EST sequences $(1,774 \mathrm{bp})$. Gene-specific primers were designed and a 1,657-bp cDNA was amplified from cv. Morex and cloned into the pGEMT-Easy vector (Promega Corp., Madison, WI, U.S.A.). The cloning of the other three HvUGT required a combination of 5'-RACE following the kit provided by Invitrogen (Carlsbad, CA, U.S.A.) and homology to rice UGT.

The cloning of a full-length cDNA corresponding to Contig13248_at progressed in four steps. First, a 407-bp-long RACE fragment was obtained, out of which 306 bp was a new segment of the cDNA. Based on the sequence of a rice UGT gene showing high similarity (NM_001058779), primers corresponding to $\mathrm{N}$-terminal sequences were designed and paired with two Contig13248-specific primers designed using the newly obtained 5'-RACE sequence obtained in the first step. In the second step, a 451-bp fragment was amplified. Based on sequences of this fragment, new primers for 5'-RACE were designed and a 240-bp fragment which contained the translation start codon was obtained in a third step. Finally, to confirm that the right $5^{\prime}$ end had been linked up with the $3^{\prime}$ end, the full-length HvUGT13248 was amplified from Morex cDNA. 
Partial cDNA sequences of 1,196 and $841 \mathrm{bp}$ were available in the database for HvUGT14077 and HvUGT19290, respectively, which were used in a National Center for Biotechnology Information BLASTn search to retrieve the closest rice homologs. This search resulted in obtaining the GenBank accession numbers NM_001050206 (for HvUGT14077) and AL663010 (for HvUGT19290) as the top hits. Primers were designed for the $5^{\prime}$ end using the rice homolog sequences and were used with gene-specific primers designed at the $3^{\prime}$ end. Extended and partial cDNA sequences, 1,465 and 1,402 bp long, were amplified for HvUGT14077 and HvUGT19290, respectively. The resulting sequences were used to design gene-specific primers for $5^{\prime}$ RACE. Upstream sequences of 170 and 217 bp for HvUGT14077 and HvUGT19290, respectively, were obtained using 5' RACE that included the translation initiation codon. Gene-specific cDNA primers were designed and full-length cDNA fragments for HvUGT14077 and HvUGT19290 were amplified from Morex, cloned into pGEMT-Easy vector, and sequenced. The resulting sequences for the four HvUGT were submitted to GenBank (Table 3).

A two-step cloning strategy based on homologous recombination in yeast was employed to construct the UGT expression vectors. First, short, synthetic adaptor DNA fragments were custom synthesized and cloned into the HindIII-NotI-digested yeast vector pYAK7, replacing the strong positive control gene PDR5. The fragments had the following basic structure: at the $5^{\prime}$ end, a HindIII site in frame with the native ATG, followed by approximately $100 \mathrm{bp}$ of $\mathrm{N}$-terminal sequence of the candidate hvUGT, separated by a $S a l$ I (or BglII) site, followed by approximately $100 \mathrm{bp}$ of C-terminal hvUGT sequence, including the STOP codon, and, finally, a NotI site at the $3^{\prime}$ end. The plasmids containing the synthetic fragments were cut with HindIII and NotI and the inserts were cloned into these respective sites in pYAK7 (Table 3 ). The resulting gap repair plasmids contained a unique $S a l$ I restriction site that was used for linearization for the subsequent yeast transformation. If a SalI site occurred within the short $3^{\prime}$ or $5^{\prime}$ region of the respective gene (as with HvUGT13248), alternatively, a $B g l$ II site was placed into the adaptor fragment to be used for linearization. Co-transformation of yeast strain YZDL112 with the UGT full-length cDNA fragments together with the linearized yeast vector containing the homologous flanking sequences led to formation of the desired expression vector by gap repair in high frequency.

The occurrence of the recombination event was tested by colony PCR (Fig. 2) and the plasmid of positive candidates was retransformed into E. coli and sequenced (Table 3, resulting plasmids). The resulting expression vectors were transformed into yeast strain YZGA515 (Table 2).

\section{Construction of the TaUGT3 expression vector and immunoblotting.}

The sequence of the TaUGT3 ORF according to FJ236328.1 with two silent changes (G993A and G561A, which destroy internal HindIII [AAaCTT] and SmaI [CCCaGG] sites) and with flanking HindIII and NotI sites was custom synthesized. The insert of the resulting plasmid (pWS2157) was cloned into pYAK7 digested with HindIII + NotI to yield the expression vector pWS2167. After reconfirmation by sequencing, this plasmid was used for transformation of yeast (Table 2). Three independent transformants were used for spotting. Western blotting of UGT with an N-terminal c-Myc tag was performed with monoclonal antibody (9E10) as previously described (Poppenberger et al 2006). Signal intensities obtained with the SuperSignal West Pico chemiluminescent substrate (Pierce, Rockford, IL, U.S.A.) were determined using a Chemidoc XRS imager (Bio-Rad, Hercules, CA, U.S.A.) and normalized for protein content (equal loading confirmed by densitometry of Coomassie-stained gel).

\section{Feeding experiment.}

For the DON feeding experiments, the trp1 strain YZDL112 was transformed with both the UGT genes (LEU2 plasmids) and with plasmid pRM561 (Mitterbauer et al. 2004), a TRP1 $A D E 2$ plasmid containing a mutant form of the gene-encoding ribosomal protein L3 (RPL $3^{W 255 C}$ ) which leads to DON insensitivity, or an empty $T R P 1$ plasmid. For a second set of experiments (Fig. 5) without the RPL3 plasmid, strain YZGA515 was used as host. In this experiment, seven independent $H v U G T 13248$ transformants were propagated at $30^{\circ} \mathrm{C}$ in liquid selective media with $2 \%$ glucose to an optical density at 600 $\mathrm{nm}\left(\mathrm{OD}_{600}\right)$ of 0.7 . Cells were harvested by centrifugation and concentrated in $4 \mathrm{ml}$ of fresh medium to an $\mathrm{OD}_{600}=2.5$. The new cultures were incubated with DON at $200 \mathrm{mg} / \mathrm{liter}$ (by adding stock dissolved in $\mathrm{H}_{2} \mathrm{O}$ at $10,000 \mathrm{mg} / \mathrm{liter}$ ) at first; after $2 \mathrm{~h}$, the concentration was raised to $500 \mathrm{mg} / \mathrm{liter}$. The experiment was stopped after $24 \mathrm{~h}$. Samples of $100 \mu \mathrm{l}$ were taken at different time points. Supernatant and pellet were separated by centrifugation. The pellet was washed twice in chilled $\mathrm{H}_{2} \mathrm{O}$ and extracted by addition of 1,250 $\mu$ l of $\mathrm{MeOH}: \mathrm{H}_{2} \mathrm{O}$ (80:20), freezing at $-80^{\circ} \mathrm{C}$ for $15 \mathrm{~min}$, and sonification on ice for another $15 \mathrm{~min}$. Both supernatant and $500 \mu \mathrm{l}$ of the extract after centrifugation were stored at $-20^{\circ} \mathrm{C}$ and used for $\mathrm{HPLC} / \mathrm{MS}$ analysis. After thawing, samples were diluted with $10 \%$ aqueous methanol. LC-MS/MS determination of DON and D3G was performed as shown previously (Berthiller et al. 2009a) using electrospray negative ionization on a 4000 QTrap LCMS/MS system (Applied Biosystems, Foster City, CA, U.S.A.). The dilution factor occurring in cell extracts was used to calculate the concentration of $\mathrm{D} 3 \mathrm{G}$ per milliliter of culture.

\section{ACKNOWLEDGMENTS}

We thank C. Erhart for excellent technical assistance, D. Lucyshyn for construction of strain YZDL112, L. Mach and B. Svoboda for purification of the anti c-Myc antibody from hybridoma (clone 9E10) supernatant, and G. Wiesenberger and J. A. Torres-Acosta for critically reading the manuscript. This work was supported by grants to G. J. Muehlbauer from the United States Wheat and Barley Scab Initiative (United States Department of Agriculture-Agricultural Research Service) and the Minnesota Small Grains Initiative, and by grants (F3708 and L255-B11) of the Austrian Science Fund FWF to G. Adam. Part of the work of G. Adam was also funded by the EU FP5 project FUCOMYR. B. Poppengerger received a DOC fellowship from the Austrian Academy of Sciences.

\section{LITERATURE CITED}

Alexander, N. J., McCormick, S. P., and Hohn, T. M. 2002. The identification of the Saccharomyces cerevisiae gene AYT1(ORF-YLL063c) encoding an acetyltransferase. Yeast 19:1425-1430.

Bai, G. H., Desjardins, A. E., and Plattner, R. D. 2001. Deoxynivalenolnonproducing Fusarium graminearum causes initial infection, but does not cause disease spread in wheat spikes. Mycopathologia 153:91-98.

Berthiller, F., Dall'Asta, C., Schuhmacher, R., Lemmens, M., Adam, G., and Krska, R. 2005. Masked mycotoxins: Determination of a deoxynivalenol glucoside in artificially and naturally contaminated wheat by liquid chromatography-tandem mass spectrometry. J. Agric. Food Chem. 53:3421-3425.

Berthiller, F., Dall'Asta, C., Corradini, R., Marchelli, R., Sulyok, M., Krska, R., Adam, G., and Schuhmacher, R. 2009a. Occurrence of deoxynivalenol and its 3-beta-D-glucoside in wheat and maize. Food Addit. Contam. 26:507-511.

Berthiller, F., Hametner, C., Krenn, P., Schweiger, W., Ludwig, R., Adam, G., Krska, R., and Schuhmacher, R. 2009b. Preparation and characterization of the conjugated Fusarium mycotoxins zearalenone- $4 O$-beta-Dglucopyranoside, alpha-zearalenol-4O-beta-D-glucopyranoside and betazearalenol-4O-beta-D-glucopyranoside by MS/MS and two-dimensional NMR. Food Addit. Contam. 26:207-213. 
Bock, K. W. 2003. Vertebrate UDP-glucuronosyltransferases: Functional and evolutionary aspects. Biochem. Pharmacol. 66:691-696.

Boddu, J., Cho, S., Kruger, W. M., and Muehlbauer, G. J. 2006. Transcriptome analysis of the barley-Fusarium graminearum interaction. Mol. Plant-Microbe Interact. 19:407-417.

Boddu, J., Cho, S., and Muehlbauer, G. J. 2007. Transcriptome analysis of trichothecene-induced gene expression in barley. Mol. Plant-Microbe Interact. 20:1364-1375.

Bowles, D., Lim, E. K., Poppenberger, B., and Vaistij, F. E. 2006. Glycosyltransferases of lipophilic small molecules. Annu. Rev. Plant Biol. 57:567-597.

Buerstmayr, H., Ban, T., and Anderson, J. A. 2009. QTL mapping and marker assisted selection for Fusarium head blight resistance in wheat-a review. Plant Breed. 128:1-26.

Desjardins, A.E. 2006. Fusarium Mycotoxins: Chemistry, Genetics, and Biology. American Phytopathological Society Press, St. Paul, MN, U.S.A.

Desmond, O. J., Manners, J. M., Schenk, P. M., Maclean, D. J., and Kazan, K. 2008. Gene expression analysis of the wheat response to infection by Fusarium pseudograminearum. Physiol. Mol. Plant Pathol. 73:40-47.

European Commission. 2006a. Commission recommendation (EC) no. 1881/2006 of 19 December 2006 setting maximum levels for certain contaminants in foodstuffs. Off. J. Eur. Union L 364/5-24.

European Commission. 2006b. Commission recommendation of $17 \mathrm{Au}-$ gust 2006 on the presence of deoxynivalenol, zearalenone, ochratoxin A, T-2 and HT-2 and fumonisins in products intended for animal feeding. Off. J. Eur. Union L 229/7-9.

European Commission. 2007. Commission recommendation (EC) no. 1126/2007 of 28 September 2007 amending Regulation (EC) no. $1881 / 2006$ setting maximum levels for certain contaminants in foodstuffs as regards Fusarium toxins in maize and maize products. Off. J. Eur. Union L 255/14-17.

Foroud, N. A., and Eudes, F. 2009. Trichothecenes in cereal grains. Int. J. Mol. Sci. 10:147-173.

Gardiner, S. A., Boddu, J., Berthiller, F., Hametner, C., Stupar, R., Adam, G., and Muehlbauer, G. J. 2010. Transcriptome analysis of the barley-deoxynivalenol interaction: Evidence for a role of glutathione in deoxynivalenol detoxification. Mol. Plant-Microbe Interact. 23:962976

Goswami, R. S., and Kistler, H. C. 2004. Heading for disaster: Fusarium graminearum on cereal crops. Mol. Plant Pathol. 5:515-525.

Guillemette, C. 2003. Pharmacogenomics of human UDP-glucuronosyltransferase enzymes. Pharmacogenom. J. 3:136-158.

Hansen, E. H., Osmani, S. A., Kristensen, C., Møller, B. L., and Hansen J. 2009. Substrate specificities of family 1 UGTs gained by domain swapping. Phytochemistry 70:473-482.

Hill-Ambroz, K. Webb, C. A., Matthews, A. R., Li, W., Gill, B. S., and Fellers, J P. 2006. Expression analysis and physical mapping of a cDNA library of Fusarium head blight infected wheat spikes. Crop Sci. 46:S15-S26.

Jackson, R. G., Lim, E. K., Li, Y., Kowalczyk, M., Sandberg, G., Hoggett, J., Ashford, D. A., and Bowles, D. J. 2001. Identification and biochemical characterization of an Arabidopsis indole-3-acetic acid glucosyltransferase. J. Biol. Chem. 276:4350-6.

Jansen, C., von Wettstein, D., Schäfer, W., Kogel, K-H., Felk, A., and Maier, F. J. 2005. Infection patterns in barley and wheat spikes inoculated with wild-type and trichodiene synthase gene disrupted Fusarium graminearum. Proc. Natl. Acad. Sci. U.S.A. 102:1689216897.

Kostelanska, M., Hajslova, J., Zachariasova, M., Malachova, A., Kalachova, K., Poustka, J., Fiala, J., Scott, P. M., Berthiller, F., and Krska, R. 2009. Occurrence of deoxynivalenol and its major conjugate, deoxynivalenol3 -glucoside, in beer and some brewing intermediates. J. Agric. Food Chem. 57:3187-3194

Lancova, K., Hajslova, J., Poustka, J., Krplova, A., Zachariasova, M., Dostalek, P., and Sachambula, L. 2008. Transfer of Fusarium mycotoxins and 'masked' deoxynivalenol (deoxynivalenol-3-glucoside) from field barley through malt to beer. Food Addit. Contam. 25:732744

Lemmens, M., Scholz, U., Berthiller, F., Dall'Asta, C., Koutnik, A., Schuhmacher, R., Adam, G., Buerstmayr, H., Mesterhazy, A., Krska, R., and Ruckenbauer, P. 2005. The ability to detoxify the mycotoxin deoxynivalenol colocalizes with a major quantitative trait locus for Fusarium head blight resistance in wheat. Mol. Plant-Microbe Interact. 18:1318-1324.

Leonard, K. J., and Bushnell, W. R., eds. 2003. Fusarium Head Blight of Wheat and Barley. American Phytopathological Society Press, St. Paul, MN, U.S.A.

Li, Y., Baldauf, S., Lim, E. K., and Bowles, D. J. 2001. Phylogenetic analysis of the UDP-glycosyltransferase multigene family of Arabidopsis thaliana. J. Biol. Chem. 276:4338-4343.

Lim, E. K. 2005. Plant glycosyltransferases: Their potential as novel biocatalysts. Chemistry 11:5486-5494.

Lim, E. K., Baldauf, S., Li, Y., Elias, L., Worrall, D., Spencer, S. P., Jackson, R. G., Taguchi, G., Ross, J., and Bowles, D. J. 2003. Evolution of substrate recognition across a multigene family of glycosyltransferases in Arabidopsis. Glycobiology 13:139-145.

Logrieco, A., Mule, G., Moretti, A., and Bottalico, A. 2002. Toxingenic Fusarium species and mycotoxins associated with maize ear rot in Europe. Eur. J. Plant Pathol. 108:597-609.

Lulin, M., Yi, S., Aizhong, C., Zengjun, Q., Liping, X., Peidu, C., Dajun, L., and Xiu-E, W. 2010. Molecular cloning and characterization of an up-regulated UDP-glucosyltransferase gene induced by DON from Triticum aestivum L. cv. Wangshuibai. Mol. Biol. Rep. 37:785-95.

Maier, F. J., Miedaner, T., Hadeler, B., Felk, A., Salomon, S., Lemmens, M., Kassner, H., and Schäfer, W. 2006. Involvement of trichothecenes in fusarioses of wheat, barley, and maize evaluated by gene disruption of the trichodiene synthase (Tri5) gene in three field isolates of different chemotype and aggressiveness. Mol. Plant Pathol. 7:449461.

McCormick, S. 2003. The role of DON in pathogenicity. Pages 165-183 in: Fusarium Head Blight of Wheat and Barley. K. J. Leonhard and W. R. Bushnell, eds. American Phytopathological Society Press, St. Paul, MN. U.S.A.

Mitterbauer, R., Poppenberger, B., Raditschnig, A., Lucyshyn, D., Lemmens, M., Glössl, J., and Adam, G. 2004. Toxin-dependent utilization of engineered ribosomal protein L3 limits trichothecene resistance in transgenic plants. Plant Biotechnol. J. 2:329-340.

Opanowicz, M., Vain, P., Draper, J., Parker, D., and Doonan, J .H. 2008. Brachypodium distachyon: Making hay with a wild grass. Trends Plant Sci. 13:172-177.

Osmani, S. A., Bak, S., and Møller, B. L. 2009. Substrate specificity of plant UDP-dependent glycosyltransferases predicted from crystal structures and homology modeling. Phytochemistry 70:325-347.

Owens, I. S., Basu, N. K., and Banerjee, R. 2005. UDP-glucuronosyltrasnferases: Gene structures of UGT1 and UGT2 families. Methods Enzymol. 400:1-22.

Pedras, M. S., Zaharia, I. L., and Ward, D. E. 2002. The destruxins: Synthesis, biosynthesis, biotransformation, and biological activity. Phytochemistry 59:579-596.

Poppenberger, B. 2003. Molecular mechanisms of resistance to Fusarium mycotoxins in plants. Doctoral thesis, University of Natural Resources and Applied Life Sciences (BOKU), Vienna.

Poppenberger, B., Berthiller, F., Lucyshyn, D., Sieberer, T., Schuhmacher, R., Krska, R., Kuchler, K., Glössl, J., Luschnig, C., and Adam, G. 2003. Detoxification of the Fusarium mycotoxin deoxynivalenol by a UDPglucosyltransferase from Arabidopsis thaliana. J. Biol. Chem. 278:47905-47914.

Poppenberger, B., Berthiller, F., Bachmann, H., Lucyshyn, D., Peterbauer, C., Mitterbauer, R., Schuhmacher, R., Krska, R., Glössl, J., and Adam, G. 2006. Heterologous expression of Arabidopsis UDP-glucosyltransferases in Saccharomyces cerevisiae for production of zearalenone-4-Oglucoside. Appl. Environ. Microbiol. 72:4404-4410.

Proctor, R. H., Hohn, T. M., and McCormick, S. P. 1995. Reduced virulence of Gibberella zeae caused by disruption of a trichothecene toxin biosynthetic gene. Mol. Plant-Microbe Interact. 4:593-601.

Sasanya, J. J., Hall, C., and Wolf-Hall, C. 2008. Analysis of deoxynivalenol, masked deoxynivalenol, and Fusarium graminearum pigment in wheat samples, using liquid chromatography-UV-mass spectrometry. J. Food Prot. 71:1205-1213.

Schothorst, R. C., and van Egmond, H. P. 2004. Report from SCOOP task 3.2.10 "Collection of occurrence data of Fusarium toxins in food and assessment of dietary intake by the population of EU member states". Subtask: Trichothecenes. Toxicol. Lett. 153:133-143.

Sewald, N., Lepschy von Gleissenthall, J., Schuster, M., Müller, G., and Aplin, R. T. 1992. Structure elucidation of a plant metabolite of 4desoxynivalenol. Tetrahedron 3:953-960.

Sexton, A. C., Minic, Z., Cozijnsen, A. J., Pedras, M. S., and Howlett, B. J. 2009. Cloning, purification and characterisation of brassinin glucosyltransferase, a phytoalexin-detoxifying enzyme from the plant pathogen Sclerotinia sclerotiorum. Fungal Genet. Biol. 46:201-209.

Shao, H., He, X., Achnine, L., Blount, J. W., Dixon, R. A., and Wang, X. 2005. Crystal structures of a multifunctional triterpene/flavonoid glycosyltransferase from Medicago truncatula. Plant Cell 17:3141-3154.

Starkey, D. E., Ward, T. J., Aoki, T., Gale, L. R., Kistler, H. C., Geiser, D. M., Suga, H., Tóth, B., Varga, J., and O’Donnell, K. 2007. Global molecular surveillance reveals novel Fusarium head blight species and trichothecene toxin diversity. Fungal Genet. Biol. 44:1191-1204.

Steiner, B., Kurz, H., Lemmens, M., and Buerstmayr, H. 2009. Differential 
gene expression of related wheat lines with contrasting levels of head blight resistance after Fusarium graminearum inoculation Theor. Appl. Genet. 118:753-764.

Stemmer, W. P. C. 1994. DNA shuffling by random fragmentation and reassembly: In vitro recombination for molecular evolution. Proc. Natl. Acad. Sci. U.S.A. 91:10747-10751.

Vigier, B., Reid, L. M., Dwyer, L. M., Stewart., D. W., Sinha, R. C, Arnason, J. T., and Butler, G. 2001. Maize resistance to Gibberella ear rot: Symptoms, deoxynivalenol and yield. Can. J. Plant Pathol. 123:99105

Vogt, T., Grimm, R., and Strack, D. 1999. Cloning and expression of a cDNA encoding betanidin 5-O-glucosyltransferase, a betanidin- and flavonoid-specific enzyme with high homology to inducible glucosyltransferases from the Solanaceae. Plant J. 19:509-519.

Walter, S., Brennan, J. M., Arunachalam, C., Ansari, K. I., Hu, X., Khan,

M. R., Trognitz, F., Trognitz B., Leonard, G., Egan, D., and Doohan, F

M. 2008. Components of the gene network associated with genotypedependent response of wheat to the Fusarium mycotoxin deoxynivalenol. Funct. Integr. Genomics 8:421-427.

AUTHOR-RECOMMENDED INTERNET RESOURCE

HarvEST database: harvest.ucr.edu 